\title{
Review
}

\section{Undesirable Events With Radiopharmaceuticals}

\author{
RAlPh SAntos-Oliveira $^{1}$ \\ ${ }^{1}$ Radiopharmacy Division, Nuclear Engineering Institute-Brazil, Rio de Janeiro, Brazil
}

\begin{abstract}
Radiopharmaceuticals are used in nuclear medicine for diagnostic and therapeutic purposes. Many adverse reactions and false positive reactions related to radiopharmaceuticals take place every day in hospitals, but most of them are not reported. It is therefore important to understand the definition of each undesirable reaction. Adverse reactions are defined as any noxious or unintended reactions to a drug, which is administered in standard doses through the proper route for the purpose of prophylaxis, diagnosis, or treatment. False positive reactions can be defined as any imaging appearance caused by undue physiological or pathological accumulation of radiopharmaceuticals. Information concerning these undesirable reactions is limited for radiopharmaceuticals. The present study intends to be a source of information that could be accessed by all nuclear medicine staff. A review of the literature from 1957 to January 2009 was carried out using the criteria of a systematic review, established by the Cochrane Collaboration, an international non-profit organization, that provides up-to-date information about the health care. The present study has revealed that radiopharmaceuticals cause adverse reactions. Six cases of adverse reactions with radiopharmaceuticals were found: 2 cases with ${ }^{18} \mathrm{~F}$-fluorodeoxyglucose (FDG) and 4 cases with technetium $99 \mathrm{~m}\left({ }^{99 \mathrm{~m}} \mathrm{Tc}\right)$. Among the 4 cases of adverse reactions with ${ }^{99 \mathrm{~m}} \mathrm{Tc}$, one subject who received ${ }^{99 \mathrm{~m}} \mathrm{Tc}$-labeled sestamibi developed anaphylactic reactions. Moreover, a total of 8 cases with false positive reactions were found with FDG. In conclusion, a worldwide effort should be made to report as many cases as possible of adverse events and false positive reactions with radiopharmaceuticals.

clinical report; adverse reaction; safety; evidence-based medicine; false positive reaction.
\end{abstract}

Tohoku J. Exp. Med., 2009, 217 (4), 251-257. (C) 2009 Tohoku University Medical Press

Lazarou et al. (1998) state that there is a high level of under-reporting of suspect adverse drug reactions (ADRs), a condition that caused over 100,000 deaths in the United States in 1994. This study was criticised for various reasons, for instance, that the death rate was calculated by applying ADR rates from studies done before 1981 to 1994 hospital admission data (Kvasz et al. 2000). Epidemiological data on adverse drug reactions are sparse and generally old. Most studies were performed 10 to 30 years ago and were relatively restricted, often confined to individual units, such as those for care of the elderly (Pirmohamed et al. 2004).

Jones (1982) had already alerted the fact that although ADRs can appear as isolated, specific clinical events that may be related to a number of factors in the patient's background and environment, in many situations it may not appear early as a detectable clinical event, but rather be clinically silent. Subclinical elevations of hepatic transaminases and blood urea nitrogen, or decreases in sperm count are typical examples of events that may or may not be detected, or are detected only by chance. With radiopharmaceuticals the situations are not different, since they are also drugs. Other descriptive characteristics of ADRs concern their acuteness or latency relative to drug exposure, their seriousness, and their frequency or incidence. The latter information, although highly desirable, is usually not well defined for most adverse reactions, except for the common ones defined in clinical trials. The incidence of rarer effects is known for just a few drugs, as only recently have epidemiological methods and studies been directed to this area.

False positive reactions become of great interest for nuclear medicine and for pharmacovigilance studies. Disease states may alter the biodistribution of radiopharmaceuticals. Also, some drugs can do the same like the analogues of somatostatin used therapeutically in carcinoid syndrome; due to a competition for the receptor sites, the use of ${ }^{111}$ In-pentetreotide results in a false negative imaging (Santos-Oliveira et al, 2008).

In any discussion on adverse reactions to radiopharmaceuticals it is frequently difficult to define what constitutes an adverse reaction (Sampson 1993). Adverse reactions have been defined as "any adverse event associated with the use of a drug in humans, whether or not considered drug

Received February 9, 2009; revision accepted for publication March 7, 2009.

Correspondence: Ralph Santos-Oliveira, Radiopharmacy Division, Nuclear Engineering Institute-Brazil, Rua Hélio de Almeida 75 Rio

de Janeiro-Rio de Janeiro, Brazil, 21941906.

e-mail: roliveira@ien.gov.br 
related: an adverse event occurring in the course of the use of drug product in professional practice; an adverse event occurring from drug overdose, whether accidental or intentional; an adverse event occurring from drug withdrawal; and any significant failure of expected pharmacologic action" (Scott 1997; Silberstein and Ryan 1996). Silberstein and Ryan (1996) proposed an alternative definition of adverse reactions to radiopharmaceuticals. According to them, "adverse drug reaction is any response to a drug which is noxious and unintended, occurring at doses used in man for prophylaxis, diagnosis, therapy of disease, or for modification of physiologic function". This is not an official definition, but is more applicable to radiopharmaceuticals.

Unlike drugs given for therapeutics purposes, radiopharmaceuticals rarely cause adverse reactions. This safety can be explained by the usually very small amount of drug injected or ingested, and also by the fact that radiopharmaceuticals are typically administered only once or a very limited number of times to any given patient (Silberstein and Ryan 1996). Nevertheless, the possibility of adverse reaction to an administered radiopharmaceutical does exist (Hesselwood and Keeling 1997). The ADRs linked to radiopharmaceuticals show just a few incidents, which however may be due to the small number of reported cases. In fact, it has been demonstrated that only $10 \%$ of possible reactions are actually reported (Keeling 1994). The great majority of these cases are not serious or life-threatening; indeed, many do not require active treatment (Keeling 1994).

Sampson (1993) classified the adverse reactions to radiopharmaceuticals into four classes: Type I, anaphylatic [similar to the description of adverse reactions Type B idiosyncratic or allergy] (Wiffen et al. 2002); Type II, cytotoxic; Type III, immune complex-mediated; and Type IV, cell mediated. This classic classification, well adapted to allergic reactions, is not entirely applicable to radiopharmaceuticals.

As an attempt to correct or make the criteria of adverse reactions more applicable to radiopharmaceuticals, an algorithm was developed (suggested) by Silbertein and Ryan (1996) and also adopted by Hesselwood and Keeling (1997) to study adverse reactions to radiopharmaceuticals. In this algorithm probabilities of causation of an adverse reaction to radiopharmaceuticals are categorised into: Not related, Conditional (Unlikely or Remote), Possible, and Probable. The last one means that a real adverse event to radiopharmaceutical occurred.

It is important to notice that a subjective interpretation of an event can be very dangerous. In order to avoid a subjective interpretation, Silberstein and Ryan (1996) stated that a significant adverse drug reaction which should be reported must include: 1 , untoward effects whether observed frequently or rarely; 2, untoward effects never before seen following administration of the radiopharmaceutical; 3 , only life-threatening or fatal reactions from drugs other than radiopharmaceuticals; 4 , reactions unanticipated from unknown pharmacologic action of a nonradioactive pharmaceutical; and 5, anaphylactoid or allergic reactions.

Estimates of adverse reaction prevalence are difficult to assess, partly because of physician/doctor ignorance of available reporting schemes. The reasons for not filling out adverse reactions reporting forms are many, among them because of the reactions' subjective aspect and of physician's anxiety about potential liability or belief that the reaction is common knowledge (Silberstein and Ryan 1996; Silberstein 1998).

A good example was the in vivo study of Turgut et al. (2006) where iodine-131 was administered to a rat to simulate diagnostic (low dose) and therapeutic (high dose) applications. A group of 20 Wistar rats were used (7-12 mo of age, body weight $>300 \mathrm{~g}$ ). The aim of their study was to establish a relationship between I-131 thyroidal uptake up-take and the $p 53$ mutation in the salivary gland. The mutation in the $p 53$ tumor suppressor gene is most frequently seen in human malignancies. According to the authors, the $p 53$ tumor suppressor gene mutation is not related to high or low-doses of iodine-131, i.e., there was no significant relationship between thyroidal uptake alterations and $p 53$ gene mutation in the salivary gland.

Recently, Santos-Oliveira et al (2008) stated that there is a considerable body of evidence that biodistribution and pharmacokinetics of radiopharmaceuticals may be altered by a variety of drugs. However in their study no single comment was made related to false positive reactions. It is important to notice that false positive reactions are so severe as any type of drug interaction. In most of cases they are more dangerous, once they are always responsible for misdiagnosis, leading to an incorrect treatment or even to an excess (unnecessary) irradiation of the patient due to the examination repetition.

\section{Methodology}

A review of the literature on drug interaction with radiopharmaceuticals and false positive reactions was carried out, using the systematic review criteria. Computerized databases for radiopharmaceuticals were searched, such as MEDLINE, EMBASE, International Pharmaceutical Abstracts and Science Citation Index, published between 1956 and 2009 (January), using "radiopharmaceuticals/drug interactions", "radiopharmaceuticals/interactions", "false positive reaction/radiopharmaceuticals" and many others as keywords. The searches were supplemented with manual searches of bibliographies of the published articles on major radiopharmacy textbooks, and in the Cochrane Database of Systematic Review. The present review of the literature uses a selection of the collected material. Controlled trials, cohort studies, case-control studies and case series in English, French, German and Portuguese were considered. The difficulty to find studies related to radiopharmaceuticaldrug interaction and false positive reactions forced the acceptance of low-quality studies (with less than 10 reported 
cases), but those provided information not found in other literature. Besides, criteria included analyses of all study reports on prospective or retrospective monitoring of drug interaction with radiopharmaceuticals. All the papers were retrieved and reviewed.

\section{Technetium - Adverse Reaction}

After the striking article by Hladik et al (1982), Spicer et al (1985) described a true adverse allergic reaction to technetium $99 \mathrm{~m}\left({ }^{99 \mathrm{~m}} \mathrm{Tc}\right)$. According to the authors, a 60-year-old white female had a comedo-type ductal carcinoma of the breast in 1980, which resulted in a left mastectomy. By April 1983 multiple lung metastases were apparent on a chest X-ray (Table 1). On 4 April 1983 she underwent a bone scan with MDP (methylene diphosphonate associated to Tc-99m), which revealed multiple metastases to thoracic and lumbar spine and right ischium. Fortyeight hours later she had a scratchy sore throat and a pruritic, raised, erythematous rash, which persisted for 3-4 days. On 16 February 1984, another MDP bone scan was performed showing new metastatic lesions in bone. Fortyeight hours later she developed sore throat and generalized maculo-papular rash which was pruritic and erythematous. She was found to have conjunctivitis and hyperemic ulcerated pharynx consistent with the diagnosis of erythema multiforme. It was also noted that the patient had been on several chemotherapy drugs and had whole brain irradiation without any report of reaction.

The observed time delay (48 $\mathrm{hr}$ post-injection) is consistent with the earlier reports (Cordova et al.1984; Sampson 1993; Silberstein and Ryan 1996; Hesselwood and Keeling 1997; Silberstein 1998), indicating a 4-24 hr or longer time lag before the development of rash. The rash development for MDP was also the most common allergic reaction reported for MDP. Likewise, Sampson (1993) reported that the most commonly used diphosphonate, MDP, accounts for the most adverse reactions to radiopharmaceuticals, which may be due to the fact that bone scanning is the commonest single nuclear medicine procedure. The symptoms with the use of MDP are dermographism, nausea, malaise, vertigo and pruritus.

Severe systemic reactions to MDP were described by
Balan et al (2003). According to the authors, a 42-year-old woman with a history of recurrent breast cancer was injected with $555 \mathrm{MBq}(15 \mathrm{mCi})$ of MDP (Table 1). Twenty-four hours later the patient felt unwell. Puffiness developed around the eyes, together with an erythematous skin rash on the trunk and around the eyes. Biochemical tests at that time, compared with those before the bone scan, suggested abnormal liver and kidney function; however, an ultrasound scan showed no alterations in both organs. The patient responded to a regime of intravenous fluids and corticosteroids, with a return to normal renal function 15 days after the bone scan and to normal liver function another 6 days later. The dermatologic manifestations resolved within 1 week. This case confirmed the other case described above and showed that adverse reactions related to radiopharmaceuticals do occur and can be very severe.

One case recently reported by Chicken et al. (2007) involved an 80-year-old woman with a 4-month history of a left breast lump (Table 1). Past medical history included untreated allergic rhinitis. She reported allergy to penicillin but not to other drugs or plasters. She was administered nanocolloidal albumin (re-constituted under sterile conditions in the hospital's radiopharmaceutical laboratory according to the manufacturer's instructions and labeled with $14.4 \mathrm{MBq}{ }^{99 \mathrm{~m}} \mathrm{Tc}$ ). A volume of $0.2 \mathrm{ml}$ of the radiocolloid was intradermally injected overlying the tumour. After 1 hour of the injection, the patient reported itching over the breast and axilla. On examination, a raised urticarial rash was noted over the upper half of the breast extending from the injection site to the axilla. There was no drop in blood pressure or oxygen saturation. A topical steroid cream was applied with rapid resolution of both itching and rash within $30 \mathrm{~min}$. A history of hypersensitivity to human albumin products is a contra-indication to the injection of nanocolloidal albumin, and this important clinical information is easily overlooked.

Anaphylactic reactions to ${ }^{99 \mathrm{~m}} \mathrm{Tc}$-labeled sestamibi were described by Mujtaba et al. (2007). According to the authors a 63-year-old white woman was injected at rest with ten millicuries (370 MBq) of ${ }^{99 \mathrm{~m}} \mathrm{Tc}$-sestamibi (Table 1). Immediately after the application, acute shortness of breath and generalised itching developed. Examination revealed

TABLE 1. Adverse Reactions to Radiopharmaceuticals.

\begin{tabular}{lccc}
\hline \multicolumn{1}{c}{ Radiopharmaceutical } & Classification* & Age/Gender** & Disease*** \\
\hline MDP associated to Tc-99m & Probable & 60 years/female & Lung metastases \\
MDP associated to Tc-99m & Probable & 42 years/female & Breast cancer \\
Nanocolloidal albumn associated to Tc99m & Probable & 80 years/female & Breast lump \\
Sestamibi Tc99m & Probable & 63 years/female & Not described \\
18- FDG & Probable & 66 years/male & Lung cancer \\
18- FDG & Possible (sarcoid reaction) & 57 years/female & Lymphoma \\
\hline
\end{tabular}

*Classification using the algorithm of Silberstein and Ryan (1996).

** Age and gender of each patient described in the case report.

*** Disease presented by the patients. 
tachypnæa, painless macroglossia, wheezing in bilateral lung fields and a nonblanching pruritic maculopapular rash. All these symptoms were presumptive of anaphylactic reactions, and epinephrine and diphenhydramine were intravenously administered, with immediate results. On the other hand, the first case of adverse reactions to ${ }^{99 \mathrm{~m}}$ Tc-labeled sestamibi manifested erythema multiforme (Thomson and Allman 2001).

\section{Adverse Reaction To Fluorodeoxyglucose}

In recent years PET scans with ${ }^{18}$ F-fluorodeoxyglucose (FDG) have played an increasingly important role in the evaluation of the response to induction chemotherapy, as well as in the detection of primary tumour and metastatic lesions in several malignancies (Weber et al. 2003; Maeda et al. 2005; Lindsay et al. 2007). In one reported case a 66 year-old man was referred to the hospital for investigation of an abnormal shadow measuring $5.2 \mathrm{~cm}$ in diameter in the left upper lung field on a chest X-ray (Maeda et al. 2005). The patient was eventually diagnosed with lung cancer classified as clinical stage IIIA (T2N2M0) and underwent induction chemotherapy with paclitaxel. On the other hand, an FDG-PET scan prior to the chemotherapy demonstrated accumulation only in the tumour and not in the mediastinal node. This result led to suspicion that the disease had progressed to N3 lesions. Hence the patient underwent a biopsy of the right supraclavicular lymph node and the mediastinal lymph nodes by mediastinoscopy. All dissected lymph nodes showed sarcoid reactions and no tumour cells were found to be pathological. The patient had no clinical symptom of sarcoids and the chest X-ray did not show streaks and/or spots. The conclusion was that a sarcoid reaction of the mediastinal lymph nodes probably led to the abnormal accumulation of FDG without tumour metastasis. Therefore, in patients with FDG-PET positive results, it is necessary to verify lymph node pathologically using mediastoscopy, because this type of adverse reaction (sarcoid reaction) may occur and change the clinical conduction (Maeda et al. 2005).
Another case of sarcoid reaction occurred with a 57-year-old female patient with a history of diffuse large B-cell lymphoma, involving the lungs and spleen. This patient received 8 courses of CHOP (cyclophosphamide, doxorubicin, vincristine, prednisone) and rituxan therapy. After 4 months she underwent a PET-CT scan that showed increased uptake in the spleen. This patient underwent a splenectomy due to the suspicion of recurrence. The results of the pathology showed noncaseating granulomas consistent with sarcoid-like reaction.

These two cases show the importance of the sarcoidreaction and the importance of a pathological analysis in case of suspicion (Reilly et al. 2007). All the cases described above are summarised in Table 1 and were classified using the algorithm described by Silberstein and Ryan (1996).

\section{False Positive Reactions}

A case of false positive uptake, described by Iwasaki et al. (2006) with a 57-year-old woman, demonstrated that fused FDG-PET and PET/CT images performed to analyse pulmonary suture abscess can be confused with lung cancer, because the abscess, as an inflammatory process, increase the uptake of FDG by the lesion (Table 2). During the analysis, the scan data can be misunderstood as a lung cancer. A report made by Aide et al. (2005) described a false-positive uptake of FDG in three patients related to foreign body reaction. According to the authors remote mesh prostheses can induce FDG uptake because of persistent foreign body reaction. Consequently, in each scan an unexpected pelvic focus was noticed on FDG-PET. The authors confirmed that the medical history of patients should be carefully reviewed to avoid false-positive results.

Hurwitz (2003) described a 54-year-old woman with treated carcinoma of the breast (Table 2). There was spontaneous rupture of an implant placed previously as part of breast reconstruction. An FDG-PET scan was carried out and disclosed intense uptake in these nodes. Lymph nodes biopsy, however, demonstrated benign inflammatory reaction and no recurrence of malignancy. A false-positive PET

TABLE 2. False Positive Reactions with Radiopharmaceuticals.

\begin{tabular}{clll}
\hline Radiopharmaceuticals & Age/Gender* & \multicolumn{1}{c}{ Diagnosis** } & \multicolumn{1}{c}{ Misdiagnosis*** } \\
\hline 18-FDG & 57 years/female & Pulmonary suture abscess & Lung cancer \\
18-FDG & Not specified & Foreing body & Pelvic cancer \\
18-FDG & 54 years/female & Inflammation & Lymphoma \\
18-FDG & 73 years/male & Vocal cord paresis & Vocal cord cancer \\
18-FDG & Not specified & Hip arthroplasty & Bone cancer of tongue tumour \\
18-FDG & 23 years $/$ male & Treated tumour of tongue & Recurrence of tongue tumour \\
18 -FDG & 36 years/male & Treated tumour of tongue & Recurrence of tongue tumour \\
18-FDG & 37 years/male & Treated tumour of tongue & Recurrence of tongue tumour \\
\hline
\end{tabular}

* Age and gender of each patient described in the case report;

** Correct diagnosis of the disease;

*** Related misdiagnosis due to the false positive reaction. 
scan may occur when FDG is taken up in a ruptured breast implant and data should be carefully analysed in the relevant cases.

Other case of false-positive reaction was described by Modi et al. (2005) with a 73-year-old man that received Teflon to treat vocal cord paresis and showed abnormal uptake of FDG during a PET scan (Table 2). According to the authors the false-positive response was due a foreign body reaction (as described above) related to Teflon.

Another important case of false-positive reaction is related to the hip arthoplasty. A retrospective and prospective study conduced by Hongming et al. (2002) with nine patients and 710 patients respectively, concluded that, following hip arthroplasty, non-specifically increased FDG uptake around the head or neck of the prosthesis persists for many years, even in patients without any complications. Therefore, to minimize the number of false-positive results with PET studies, caution should be exercised when interpreting FDG uptake around the head or neck portion of prostheses.

Ariji et al. (2009) described recently three cases of false positive reactions related to 18-FDG. Three Japanese men of 23-37 years old, with treated tongue câncer, showed positive accumulation of 18-FDG at the end of treatment. Moreover, there was no sign or symptom of inflammation. Therefore, no single relation with a foreing body or infflammation reaction can be made. The authors associated the cases to ill-balanced high activity of the lingual muscles after definitive radiotherapy. These cases are summarized in Table 2.

\section{Statistical Data-Base}

After all the reported cases described above a great number of studies were developed to certify the real incidence of adverse reactions to radiopharmaceuticals. Silberstein and Ryan (1996), studying a total of 783,525 injections of radiopharmaceuticals in a prospective 5-year study, found 18 adverse reactions to radiopharmaceuticals. None was severe enough to cause hospitalisation. It means that adverse reactions to radiopharmaceuticals are quite uncommon, occurring with a prevalence of $2.3 / 10^{5}$ $(0.0023 \%)$. The $95 \%$ confidence limits for these reactions are $0.1-11.7$ per 100,000 injections. The study also verified the interventional pharmaceuticals (not tracers) used in nuclear medicine and found out a prevalence of 5.9/10 which means that they are also quite safe. An interesting aspect of this study was that 10 of the 18 adverse reactions reported were rashes. It is therefore conceivable that most adverse reactions are in truth of the allergic type, or, to be more specific, type I reactions, as described by Sampson (1993). The adverse reactions were generally mild and selflimiting, and none of them was sufficiently serious to require hospital admission.

A second study conducted by Hesslewood and Keeling (1997) studied 71,046 radiopharmaceutical administrations in Europe from January to December 1996. Only 18 reports of adverse reactions were received, five of which are considered to be of a vasovagal nature, but by the algorithm of Silberstein and Ryan (1996) are not considered an adverse reaction. Of the remaining 13 reports, eight were categorised as either possible or probable, with the algorithm of Silberstein and Ryan (1996). Thus if all 18 reports are included in the calculation, a prevalence of 25 (95\% confidence limits: $13-37$ ) events per $10^{5}$ administrations was obtained. If the five events described as vasovagal are excluded, this figure drops to 18.2 (95\% confidence limits: 8 -28) events per $1 \times 10^{5}$ administrations, and if only the reactions categorised as possible or probable are included the prevalence becomes 11 (95\% confidence limits: 3.3-19.2) per $1 \times 10^{5}$ administrations. The results of this study are slightly higher than those of the study of Silberstein and Ryan (1996). However, the effects noted were generally mild and self-limiting, and none was sufficiently serious to require admitting the patient to hospital, like the Silberstein and Ryan (1996) had noted.

A retrospective and prospective study carried out from 1994 to 1997 by Silberstein (1998) with 22 PET centres out of approximately 65 PET facilities across the USA revealed 33,925 radiopharmaceutical administrations without an adverse reaction. The total prospective number of administered dosages recorded by the participants was 47,876. The total number of administered doses of PET radiopharmaceuticals studied for adverse reaction was, therefore, 81,801 . In no case did an adverse reaction occur (95\% confidence limits: $0-3.7 \times 10^{-5}$ ). In addition, 3,265 non-radioactive interventional drugs were given with no serious adverse reactions. This study provided a denominator to, and confidence limits around the remarkable absence of adverse reactions to PET radiopharmaceuticals, primarily ${ }^{18} \mathrm{~F}$-fluorodeoxyglucose, but also ${ }^{11} \mathrm{C}-\mathrm{CO}_{2},{ }^{11} \mathrm{C}$-methionine, ${ }^{13} \mathrm{~N}-\mathrm{NH}_{3}$, and ${ }^{15} \mathrm{O}-\mathrm{H}_{2} \mathrm{O}$.

The data of the $27^{\text {th }}$ Report on Survey of Adverse Reaction to Radiopharmaceutical (2006) were based on responses to questionnaires sent to nuclear medicine institutions (Kusakabe et al. 2006). Out of 1,220 institutions, 968 replied. Sixteen cases of adverse reactions were reported. A total of 1,277,906 radiopharmaceutical administrations were reported. The incidence of adverse reactions per 100,000 cases was 1.3 (95\% confidence limits: $3-15$ ).

\section{Treatmet of Adverse Reaction to RADIOPHARMACEUTICALS}

Although the great majority of radiopharmaceutical adverse reaction reactions require little or no treatment, Sampson (1993) suggested a route to be followed. In most cases, reassurance of the patient is all that is necessary. For persistent, unpleasant allergic-type reactions, antihistamines such as promethazine and cinnarizine are useful. For mild reactions presenting nausea and vomiting dopamine antagonists (metoclopramide), anticholinergics (hyoscine) and antihistamines can be used. For the more severe reactions, corticosteroids may be employed. For cases of 
severe reactions associated with bronchospasm, treatment should commence immediately with oxygen, epinephrine (adrenaline) at $1: 1,000$ (0.3 to $0.7 \mathrm{~mL}$ intramuscular), steroids such as hydrocortisone (200 mg intravenous) and aminophylline $(5 \mathrm{mg} / \mathrm{kg}$ ) by slow intravenous drip. In rare cases of anaphylactic cardiovascular collapse where there may be gross vasodilation and loss of plasma, it is essential to administer oxygen, intramuscular epinephrine and colloidal plasma volume restorers.

\section{Conclusion}

In the light of what has been shown the adverse reactions to radiopharmaceuticals, as is the case with any medicament, should be better documented and reported. This information should be worldwide disseminated, as sometimes the physician in charge of the exam is not aware that an adverse reaction is taking place. As nuclear medicine is in most cases applied to seriously ill patients, in particular those with cancer, inevitably some psychosomatic reactions are to be expected, and it is sometimes difficult for the physician to identify legitimate organic reactions.

Only few studies were made in this topic, despite that the amount or frequency of radiopharmaceuticals in use nowadays is so high. It seems impossible that the reported cases covered all that truly happened. This is a clear justification for investigations on undesirable events with radiopharmaceuticals to continue in earnest.

\section{References}

Aide, N., Deux, J.F., Peretti, I., Mabille, L., Mandet, J., Callard, P. \& Talbot, J.N. (2005) Persistent foreign body reaction around inguinal mesh prostheses: a potential pitfall of fdg pet. Am. J. Roentgenol., 184, 1172-1177.

Ariji, Y., Fuwa, N., Kodaira, T., Tachibana, H., Nakamura, T., Satoh, Y. \& Ariji, E. (2009) False-positive positron emission tomography appearance with $18 \mathrm{~F}$-fluorodeoxyglucose after definitive radiotherapy for cancer of the mobile tongue. $\mathrm{Br}$. J. Radiol., 82, e3-7

Balan, K.K., Choudhary, A.K., Balan, A. \& Wishart, G. (2003) Severe systemic reaction to 99Tc-methylene diphosphonate: a case report. J. Nucl. Med. Tech., 31,76-78.

Cordova, M.A., Hladik, W.B. \& Rhodes, B.A. (1984) Validation and characterization of adverse reactions to radiopharmaceuticals. Noninv. Med. Imag., 1, 17-24.

Chicken, D.W., Mansour, R., Ell, P.J. \& Keshtgar, M.R.S. (2007) Allergy to technetium-labelled nanocolloidal albumin for sentinel node identification. Ann. R. Coll. Surg. Engl., 89, 1-2.

Hladik, W.B., Nigg, K.K. \& Rhodes, B.A. (1982) Drug-induced changes in the biological distribution of radiopharmaceuticals. Semin. Nucl. Med., 2, 184-218.

Hesselwood, S.R. \& Keeling, D.H. (1997) Frequency of adverse reactions to radiopharmaceuticals in europe. Eur. J. Nucl. Med., 24, 1179-1182.

Hongming, Z., Chacko, T.K., Hickeson, M., Stevenson, K., Qi, F., Ponzo, F., Garino, J. \& Alavi, A. (2002) Persistent non-specific fdg uptake on pet imaging following hip arthroplasty. Eur. J. Nucl. Med., 29, 1328-1333.

Hurwitz, R. (2003) F-18 fdg positron emission tomographic imaging in a case of ruptured breast implant: inflammantion or recurrent tumor? Clin. Nucl. Med., 28, 755-756.

Iwasaki, T., Kakagawa, K., Katsura, H., Nakane, S., Kawahara, K. \& Fukuda, H. (2006) Pulmonary suture abscess with false- positive 18-f-fluorodeoxyglucose positron emission scan mimicking lung cancer recurrence. Jpn. J. Thorac. Cardiovasc. Surg., 54, 351-355.

Jones, J.K. (1982) Adverse drug reactions in the community health setting:approaches to recognizing, counseling, and reporting. Fam. Community Health, 5, 58-67.

Keeling, D.H. (1994) Adverse reactions and untoward events associated with the use of radiopharmaceuticals. In: Sampson, CB, ed, Textbook of radiopharmacy theory and practice. Yverdon: Gordon and Breanch Sciece Publishers, p 285-295.

Kusakabe, K., Okamura, T., Kasagi, K., Komatani, A., Sato, Y., Matsuda, H., Maruno, H., Subcommittee for Safety Issues of Radiopharmaceuticals, Medical Science, \& Pharmaceutical Committee, Japan Radioisotope Association. (2006) $27^{\text {Th }}$ Report on Survey of Adverse Reaction to Radiopharmaceutical (The $30^{\text {Th }}$ survey in 2004). Kaku Igaku, 43, 23-25. (In Japanese)

Kvasz, M., Allen, I.E., Gordon, M.J, Ro, E.Y., Estok, R., Olkin, I. \& Ross, S.D. (2000) Adverse drug reactions in hospitalized patients: a critique of a meta-analysis. Medscape General Medicine 2, E3.

Lazarou, J., Pomeranz, B.H. \& Corey, P.N. (1998) Incidence of adverse drug reactions in hospitalized patients- a meta-analysis of prospective studies. JAMA, 279, 1200-1204.

Lindsay, M.J., Siegel, B.A., Tunis, S.R., Hillner, B.E., Shields, A.F., Carey, B.P. \& Coleman, R.E. (2007) The National Oncologic PET Registry: Expanded Medicare Coverage for PET under coverage with evidence development. Am. J. Roentgenol., 188, 1109-113.

Maeda, J., Ohta, M., Hirabayashi, H. \& Mtsuda, H. (2005) False positive accumulation in $18-\mathrm{F}$ fluorodeoxyglucose positron emission tomography scan due to sarcoid reaction following induction chemotherapy for lung cancer. Jpn. J. Thorac. Cardiovasc. Surg., 53, 196-198.

Mujtaba, B., Adenaike, M., Yaganti, V., Mujtaba, N. \& Jain, D. (2007) Anaphylatic reaction to tc-99m sestamibi (cardiolite) during pharmacologic myocardial perfusion imaging. J. Nucl. Cardiol., 14, 256-258.

Modi, D., Fulham, M.J., Mohamed, A. \& Havas, T.E. (2005) Markedly increased fdg uptake in a vocal cord after medialization with teflon: pet/ct findings. Clin. Nucl. Med., 30, 45-47.

Pirmohamed, M., James, S., Meakin, S., Green, C., Scott, A.K., Walley, T.J., Farrar, K., Park, B.K. \& Breckenridge, A.M. (2004) Adverse drug reaction as cause of admission to hospital: prospective analysis of 18820 patients. BMJ, 329,15-19.

Reilly, T.B., Schuster, D.M., Starsiak, M.D., Kost, C.B. \& Halkar, R.K. (2007) Sarcoid-like reaction in the spleen following chemotherapy for non-hodgkin's lymphoma. Clin. Nucl. Med., 32, 569-571.

Sampson, C.B. (1993) Adverse reactions and drug interactions with radiopharmaceuticals. Drug Saf., 8, 280-294.

Santos-Oliveira, R., Smith, S.W. \& Carneiro-Leão, A.M.A. (2008) Radiopharmaceuticals drugs interactions: a critical review. An. Acad. Bras. Cien., 80, 665-675

Scott, J.R. (1997) Canadian adverse reaction reporting program for radiopharmaceuticals summary, january 1989-January 1991. Society of Nuclear Medicine of Canada, Bulletin 7, ed. Spring, sp.

Silberstein, E.B. (1998) Prevalence of adverse reactions to positron emitting radiopharmaceuticals in nuclear medicine. Pharmacopeia Committee of the Society of Nuclear Medicine. $J$. Nucl. Med., 39, 2190-2192.

Silberstein, E.B. \& Ryan, J. (1996) Prevalence of adverse reactions in nuclear medicine. Pharmacopeia Committee of the Society of Nuclear Medicine. J. Nucl. Med., 37, 185-192.

Spicer, J.A., Preston, D.F. \& Stephens, R.L. (1985) Adverse allergic reaction to technetium-99m methylene disphosphonate. $J$. Nucl. Med., 26, 373-374.

Thomson, L.E. \& Allman, K.C. (2001) Erythema multiforme reac- 
tion to sestamibi. J. Nucl. Med., 42, 534.

Turgut, B., Ozdemir, O. \& Erselcant, T. (2006) Evaluation of the p53 Tumor Suppressor gene mutation in the normal rat salivary gland tissue after radioiodine application: an experimental study. Adv. The., 23, 456-468.

Weber, W.A., Petersen, V., Schimdt, B., Tyndale-Hines, L., Link, T., Peschel, C. \& Schwaiger, M. (2003) Positron emission tomog- raphy in non-small-cell lung cancer: prediction of response to chemotherapy by quantitative assessment of glucose use. $J$. Clin. Oncol., 21,2651-2657.

Wiffen, P., Gill, M., Edwards, J. \& Moore, A. (2002) Adverse drug reactions in hospital patients - a systematic review of the prospective and retrospective studies. Bandolier June. 